\title{
Novel Use of Ultrasound in Evaluation of Adenoid Hypertrophy in Children
}

\author{
Manisha Jana ${ }^{1}$ (D) Arun Kumar Gupta ${ }^{1}$
}

Received: 19 August 2020 / Accepted: 3 September 2020 / Published online: 10 September 2020

(C) Dr. K C Chaudhuri Foundation 2020

Adenoid hypertrophy in children may have protean manifestations. The symptoms result from obstruction of the nasopharyngeal airway; and may range from snoring, sneezing, repeated attacks of upper respiratory infection and serous otitis media. Chronic obstruction leads to mouth-breathing, and resultant changes in the growing facial skeleton; thereby resulting in the classical 'adenoid facies'. Obstructive sleep apnea, and chronic hypoxia may eventually lead to right heart dysfunction and pulmonary arterial hypertension. The normal growth of adenoids remains at its peak during 6-8 y; which is followed by physiological involution during adolescence $[1,2]$. The usual age of presentation of adenoid hypertrophy also parallels this time course. The causative factors implicated in the hypertrophy include allergy, atopy, frequent infections and chronic sinusitis.

Establishing a clinical diagnosis of adenoid hypertrophy may not be difficult in the eyes of a seasoned pediatrician; but assessing the severity and taking a decision of surgery more often requires some objective evidence. Lateral radiograph of the neck has remained the most widely used investigation for measurement of adenoid thickness and nasopharyngeal airway compromise $[1,2]$. Adenoid/ nasopharynx $(\mathrm{A} / \mathrm{N})$ ratio is taken as a marker of the severity of nasopharyngeal airway obstruction by adenoid hypertrophy [3]. However, the measurements should be performed on a proper lateral projection; as any degree of obliquity can affect the measurement. $3 \mathrm{D}$ evaluation of the airway by cone beam computed tomography (CBCT) has also been used by some researchers for more accurate evaluation of adenoid hypertrophy and its effects on airway [4]. However, both these modalities come with the drawback of exposure to ionizing radiation. This is even

Manisha Jana

manishajana@gmail.com

1 Department of Radiodiagnosis, All India Institute of Medical Sciences, New Delhi 110029, India more important for a child who has to undergo repeated imaging. Nasopharyngoscopy provides an accurate estimation of airway compromise; but it is invasive.

Ultrasound is a versatile imaging modality that has been used in evaluation of the larynx, cervical part of trachea and even the palatine tonsils. However, it has not been used to evaluate adenoids till date. The article by Wang et al. in the present issue of Indian Journal of Pediatrics describes the reliability of ultrasound in detection of adenoid hypertrophy [5]. The authors conducted ultrasound examinations in 1898 children of 3-12 y age group; and assessed the size, appearance and vascularity. They found that the gland has its maximum thickness at the age of $6 \mathrm{y}$. In a subset of 133 children who underwent adenoidectomy; the authors correlated the ultrasound estimated adenoid thickness (AUT) with radiographic measurement ( $\mathrm{A} / \mathrm{N}$ ratio) and nasopharyngoscopic measurement of adenoid-posterior nasal occlusion. They found a good correlation of AUT with the latter two parameters. They concluded that an AUT of $>6 \mathrm{~mm}$ can be taken as an indication for surgical adenoidectomy.

The application of ultrasound in evaluation of adenoid hypertrophy has the potential to substantially modify the present diagnostic algorithm; as this does not come with the risk of ionizing radiation. However, as applicable to ultrasonography anywhere else; this is operator dependent and has a longer learning curve. Nevertheless, these drawbacks can be overcome by operator training in adopting proper technique of scanning. Since this is a new area of imaging, strict adherence to imaging protocols and methods of measurement is a must.

\section{Compliance with Ethical Standards}

Conflict of Interest None. 


\section{References}

1. Orji FT, Ezeanolue BC. Evaluation of adenoidal obstruction in children: clinical symptoms compared with roentgenographic assessment. J Laryngol Otol. 2008;122:1201-5.

2. Gangadhara Somyaji KS, Rajeshwari A, Jain M. Significance of adenoid nasopharyngeal ratio in the assessment of adenoid hypertrophy in children. Res Otolaryngol. 2012;1:1-5.

3. Talebian S, Sharifzadeh G, Vakili I, Golboie SH. Comparison of adenoid size in lateral radiographic, pathologic, and endoscopic measurements. Electron Physician. 2018;10:6935-41.
4. Oh KM, Kim MA, Youn JK, Cho HJ, Park YH. Three-dimensional evaluation of the relationship between nasopharyngeal airway shape and adenoid size in children. Korean J Orthod. 2013;43:160-7.

5. Wang Y, Jiao H, Mi C, Yang G, Han T. Evaluation of adenoid hypertrophy with ultrasonography. Indian J Pediatr. 2020. https:// doi.org/10.1007/s12098-020-03203-4.

Publisher's Note Springer Nature remains neutral with regard to jurisdictional claims in published maps and institutional affiliations. 